\title{
Fault Detection of Fuel Systems Using Polynomial Regression Profile Monitoring
}

\author{
Mahmoud Awad \\ American University of Sharjah, UAE \\ miawad@aus.edu
}

\begin{abstract}
Anomaly detection is the characterization of a normal behavior of a system or process and identification of any deviation from such normal behavior. Anomaly detection of critical systems provides an important financial and client competitive advantage since it gives the decision-maker lead-time and flexibility to manage the health of the system. Fuel systems are complex and mission critical systems that require high operational availability because of the high costs associated with the services they provide. In complex systems, it is not uncommon to monitor a quality-related response which relies on the functional form between several variables using a non-linear relationship. We present in this paper a new monitoring framework for smart fuel systems utilizing outlying observations detection and monitoring using ccharts. The traditional control charts based on the Hotelling's $T^{2}$ statistic were deficient in detecting SFS anomalies and a new approach was necessary to isolate faulty profiles. The proposed methodology requires a simple quality performance test that can be performed once assembly is completed to assure readiness for client use or completion of a job. The results were tested and validated using scaled data that mimic an actual system. The methodology presented in this paper is scalable and can be applied to a wide range of systems to assess their health from an inspection check to anticipate and avoid failures.
\end{abstract}

Keywords—Profile monitoring; fault detection; fuel systems; SPC

\section{INTRODUCTION}

With the advent of new technologies, industrial systems in general are becoming more complex with the need for better quality always increasing. Moreover, technology advancements increased dramatically the amount of data measured in industrial processes. Anomaly detection is the extraction of data underlying normal structural and identification of departures from such normal structures. This can be done by defining healthy sets or classes for the different regimes of operation of the system, and identifying anomalies as points diverging from the normal classes or clusters. The use of fault or anomaly detection is a key strategy used by manufacturers to avoid major field failures, improve maintenance, move from unscheduled maintenance to condition-based maintenance, and decrease the total cost of ownership of these systems. Fault detection has been applied successfully in many fields, including aerospace, electronics, medicine, automotive, and others [1] [2] [3] [4] [5].

Fuel systems are engineered systems designed to supply fuel from a storage area to power generating systems like engines, generators, boilers, etc. They are used in many industrial applications such as power generators, automobiles, and aircrafts to supply fuel (gas or diesel) to internal combustion engines which will burn the fuel to generate power. The cost of building, operating, and maintaining such systems is high and may range from tens of thousands to millions of dollars depending on the application. In some cases, depending on demand, fuel systems are highly utilized with semi-continuous operation and have to go through tight and time consuming scheduled maintenance.

In essence, fuel systems are electro-mechanical systems with a motor-pump backbone system and have to operate in a harsh environment such as high temperature, continuous operation, contamination, and water presence in fuel. A fuel system consists of an electric motor, fuel pump, fuel filter, fuel rails, pressure regulator, pulse damper, and injection valves. The fuel pump runs constantly to supply the engine with sufficient fuel at a desired pressure $(P)$ and flow rate $(F R)$ in all operating conditions. In old fuel systems, pumps are constantly running regardless of the load and excess fuel is allowed to flow back to the fuel tank. Smart fuel systems (SFS) however, have a closed loop system where the speed of the motor changes according to load and enough fuel is pumped. The electric power is fed to the motor via transistors mounted on a printed wire assembly (PWA) known as motor driver while the switching of these transistors is controlled (when and how long) using a motor controller PWA. The motor controller is a pulse width modulated (PWM) controller where proportional voltage is controlled by controlling the PWM duty cycle. A power supply PWA is another essential element of SFS systems with main function of supplying and regulating power to motor controller and driver. When the motor starts spinning, electric energy is 
converted into mechanical through a hydraulic pump which is used to move fuel. The minimum power $P_{\min }$ required to drive fuel from point 1 to point 2 can be expressed as:

$$
P_{\min }=F R_{f} \cdot\left[\frac{v \cdot \alpha \cdot\left(P_{2}^{2}-P_{1}^{2}\right)}{2}+v \cdot\left(P_{2}-P_{1}\right)\right]
$$

where $F R_{f}$ is fuel flow rate, $v$ is fuel specific volume, $\alpha$ is fuel isothermal compressibility, $P_{1}$ and $P_{2}$ are pressure at point 1 and point 2 respectively.

Motor load and speed is variable, and controlled by the amount of current and voltage dumped into the motor. The system is serial in nature, and the overall efficiency is estimated as the product of the individual efficiencies of the power supply, motor driver, motor controller, motor, pump, and actuation system. The motor current $I_{\text {motor }}$ drawn can be represented as a function of some of the system variables as shown in equation 2.

[6]

$$
I_{\text {motor }}=\frac{\tau_{\text {motor }} \cdot \omega_{\text {motor }}}{\mu_{\text {motor }} \cdot V}=\frac{\Delta P_{f} \cdot F R_{f}}{\mu_{\text {pump }} \cdot \mu_{\text {motor }} \cdot V}
$$

where $\tau_{\text {motor }}$ and $\omega_{\text {motor }}$ are output motor torque and speed respectively, $\Delta P_{f}$ is the delta pressure across the pump inlet and outlet, $V$ is voltage supply, $\mu_{\text {motor }}$ and $\mu_{\text {pump }}$ are motor and pump efficiencies respectively. Keep in mind that equation 2 is not inclusive to all variables impacting motor current such as duty cycle of the transistors, pump irreversibility, and other losses.

The SFS is part of a more complicated system used for power generation. Depending on the location where the failure happens, the cost of failing to deliver required service can be extremely high especially if the operation is in remote area. Due to the complexity and nature of nonlinear data describing SFS's, researchers and practitioners attempted to develop additional measurement hardware such as current and vibration to possibly monitor such systems and detect any faults early on [7] [6]

The SFS is typically assembled and goes through several in-process and assembly tests. Once in operation, the SFS system goes through challenging operational schedules and harsh environment. Due to design and manufacturing defects, a simple and short end-of-line (EOL) quality test is conducted where the SFS is run with different loads and speeds. During this EOL quality test, several variables are monitored and used to decide whether to ship the system to field or send it for repair. It is not uncommon to pass this quality test and then fail in the field causing a long downtime and expensive system repair. One particular failure mode of interest is SFS shutdown due to excessive drawn current. Although the signature or profile of such failure mode (excessive drawn current) is unique, several causes may lead to the same profile. Examples of causes include transistors failure, excessive pump friction, environmental abuse, and motor winding insulation failure. The dominant failure mechanism is thermo-mechanical stress of the insulated gate bipolar transistor (IGBT) causing it to degrade over time and finally fail. The amount of data collected from any fuel system during EOL quality test is a function of the number of data channels/variables, sampling rate, and test duration. Number of channels is typically in the range of 10-30 channels, sampling rate is 10 $\mathrm{kHz}$, and test duration is in the range of 10-20 minutes generating large amount of measurement observations.

Although motor current $I_{\text {motor }}$ is known to be a great indicator of over or under load situation caused by failures in any of the major components of the system [8] [9] [7], it is not an obvious anomaly detector by itself. Figure 1 below shows measured motor current profile of a good (healthy-HS) and a system that passes the EOL quality test and failed in the field (faulty-FS), it is clear that the two profiles are hardly distinguishable.

Due to proprietary reasons, the original measurement data were partially coded in this article without impacting the conclusions and method presented. The data includes observations from known healthy SFS systems, SFS systems that pass EOL test and had no issues in the field, as well as SFS systems that pass the EOL test but fail in the field (faulty). The actual number of profiles and status of each profile (healthy, faulty) were kept the same as in the real-life case; however the actual input data within each profile were coded to a $[-1,1]$ range and actual current output was scaled down by dividing all observations by an integer. The purpose of this article is to propose a method to detect any latent failure(s) before going into a critical mission operation in the field using the data output of the EOL quality performance test. 


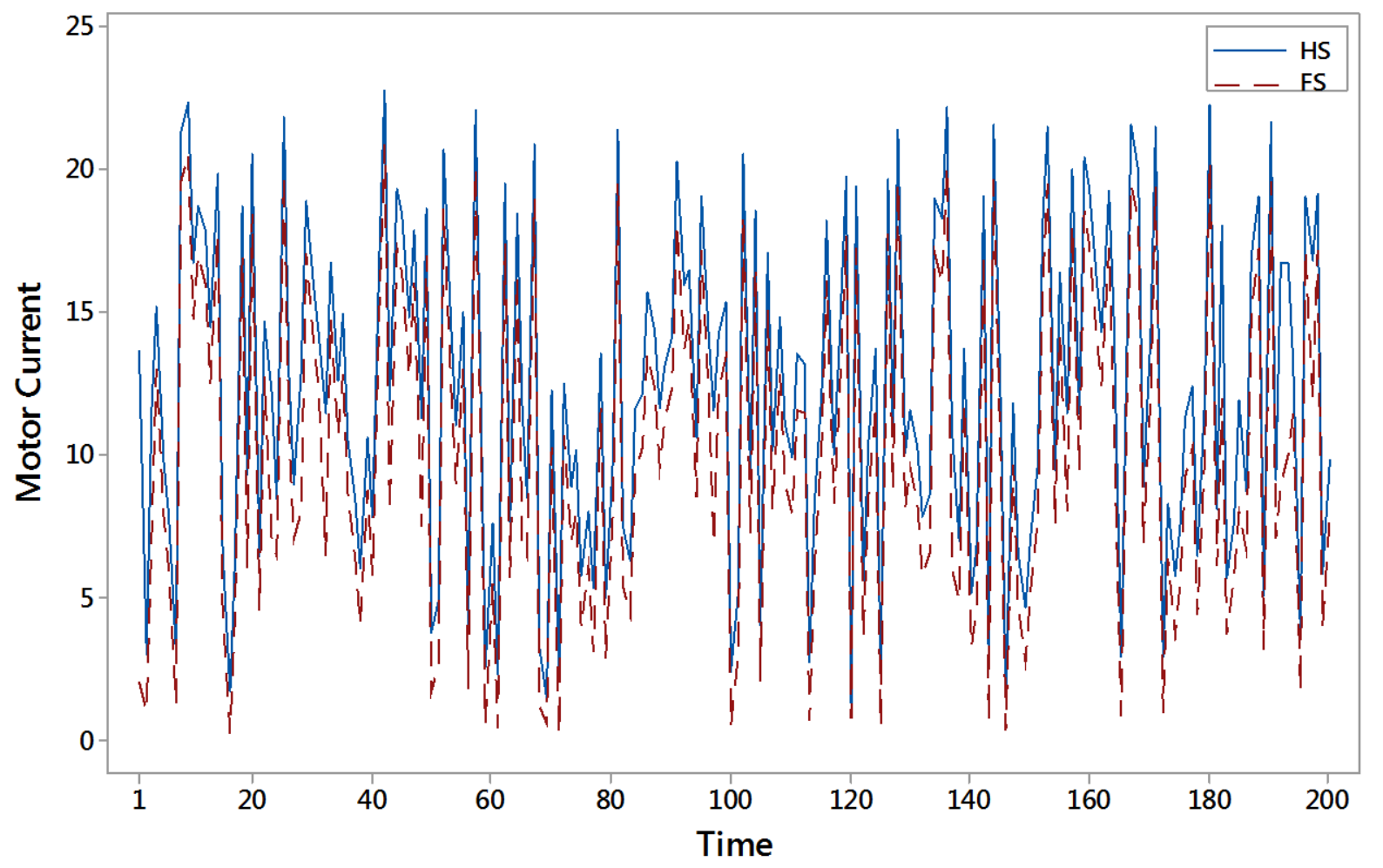

Figure 1 Motor current of healthy and faulty systems

The motivation of this paper is two folds: the wide spread usage of the electro-mechanical systems such as SFS systems, and the inability of traditional profile monitoring to identify faulty ones. As will be shown in section three, the traditional control charts based on the Hotelling's $T^{2}$ statistic was unable to detect the faulty profiles and a new method was granted. The paper is organized as follows: section two provides literature review of some of techniques used to detect system anomalies; section three demonstrates the deficiency of traditional statistical process control (SPC) methods to detect SFS anomalies and outlines the proposed methodology used to tackle the problem. Section four summarizes the results, and finally conclusions are presented in the last section.

\section{FAUlt Detection APPROACHES}

There are two major classes of approaches for fault detection: model-based approaches, and data driven-based approaches with a huge dominance of data driven approaches. Model-based approaches utilize prior knowledge of product design, loading, and physics of failures to detect any potential failures. Data-driven (DD) approaches use existing current and historical data to isolate faulty patterns of products using machine learning and statistical methods. Das et al., [10] provided a comparative evaluation of process monitoring fault detection (PMFD) approaches and concluded that there is no single strategy that can address all aspects related to process monitoring and fault detection efficiently. More detail on each approach will be provided below.

\section{A. Data Driven-Based Approaches}

Data-driven approaches are very useful for complex systems where the knowledge of the underlying physics of a system is absent or not straightforward. Several techniques are associated with DD approaches for fault management such as statistical based approaches, analytical approaches, and artificial intelligence (AI) based approaches [10] [11]. Majority of statistical based approaches utilizes statistical control charts in which the quality of a process or a system is characterized by key process variables which are monitored to detect any special cause that diverts the process or system to an "out of control" state. The AI techniques utilize either artificial neural network (ANN) or 
fuzzy logic with more dominance to the ANN approach [10]. Ge et al., [12] provided an extensive review of the nature and characteristics of different industrial processes measurement data such as high dimensionality, auto correlation, time-varying and multimode behavior, non-linearity, and non-Gaussian along with a review of the different DD process monitoring approaches suitable to handle each data type. Venkatasubramanian and Kavuri [11] broadly classified fault diagnosis methods into three general categories: quantitative model-based methods, qualitative model-based methods, and process history based methods. The third category, also known as DD based methods, do not assume any form of model information and rely only on historic process data. The same authors further classified the process history based methods into two classes: qualitative approaches such as expert systems and qualitative trend analysis (QTA), and quantitative approaches such as neural networks (NN), principal component analysis (PCA) and statistical classifiers. Severson et al., [13] provided some perspectives on progress in the design and techniques used in process monitoring systems with focus on fault detection and diagnosis (FDD) over the last twenty years. According to the same authors, types of process models employed in fault detection can be grouped under three main categories: dimensionality reduction such as principal component analysis (PCA) and partial least squares (PLS), state space models, and graphical models such as decision trees. In PCA techniques, a linear or nonlinear dimensionality reduction is done to represent original data using lower dimensional loading vectors that maximize the retained variance. When the order of measured variables is in the order of several dozens or higher, i.e. megavariate, dimensionality reduction using PCA and PLS becomes more necessary to simplify the estimation of the process parameters and improve its stability [14]. In return, the variance is used as a metric for fault detection using the Hotelling's $T^{2}$ statistic which will be introduced in SPC section below.

Since type (measurements, images, etc.), structure (cross-correlation, autocorrelation), and dimensionality (one, two, and higher dimensions) of data collected is versatile, researchers proposed modifying traditional DD techniques to handle different types and structures of data. For example, Nomikos and McGregor [15] proposed a multiway principle-component analysis (MPCA) to compress the time-varying trajectory data with high correlated variables collected in many batch processes such as production of polymers, pharmaceuticals, and biochemical. For monitoring multi- and megavariate systems whose variables present significant levels of autocorrelation three classes of approaches were proposed: control limits adjustment, embedded dynamical modeling, and data transformation techniques [16]. The first class of approaches uses the common SPC charts with control limits adjusted using analytical corrective methods such as auto-regression (AR) for simple dynamic processes. In the second class, residuals are calculated as the difference between values generated from a developed dynamic prediction model that removes autocorrelation trend and actual measured values. Assuming adequacy of dynamic model, residuals should be normally distributed with zero mean and constant variance. The deviation of residuals from this normal or healthy state is used for fault detection. Examples of such dynamic models are dynamic principal component analysis DPCA [17] [14] [18] and dynamic partial least square DPLS [19]. Finally, the third class of approaches known as multiscale multivariate SPC is based on transforming the auto-correlated time-series structure into other forms using transforming families such as wavelet transform family [20] [21] [22] [23] [24].

The DPCA approach proposed originally by Ku et al., [25] has quickly gained popularity due to its simplicity and ability to minimize the effect of autocorrelation. Rato and Reis [14] contended that although DPCA is capable of handling both the cross-and-auto-correlation nature of data, the resulting monitoring statistic used such as $T^{2}$ still present significant autocorrelation. As a result, they proposed a set of multivariate statistics (DPCA-DR) that combine DPCA and the generation of de-correlated residuals based on missing data imputation which present low auto-correlation levels. Similarly, Chiange and Brattz [26] discussed another inherent limitation of PCA and PLS related to its ability to identify and diagnose faults, especially when the abnormal situations are associated with unknown faults or multiple faults. As a result, they proposed a modified distance (DI) and modified casual dependency $(\mathrm{CD})$ metrics to incorporate the casual map with data-driven approaches to improve the proficiency of identifying and diagnosing faults. Finally Reis and Saraiva [27] proposed a heteroscedastic latent variable model approach to handle noisy data with low signal to noise ratio.

In this article, a DD based approach is utilized to detect faults of a complex fuel system before shipment to field. In this approach, a profile between motor current and several system explanatory variables such as pressure, flow rate, speed, and duty cycle is developed and used to identify any outlying observations which in return used to identify faulty systems. The next two sections provide more details on model-based and SPC fault detection approaches. 


\section{B. Model-Based Approaches}

Due to the diversity of EMSs, different approaches were utilized to detect faults of such systems depending on the type of hardware and nature of data collected. Literature is rich of research conducted to detect mechanical faults related to bearing and gearboxes using vibration signatures [28] [29] [30]. The vibration signatures are monitored using accelerometers placed close to fault sources and require processing using signal processing techniques. Due to cost and packaging issues in placing the accelerometers, some practitioners preferred using internal signatures such as motor current to overcome these inconveniences [31] [32, 33] [8] [7] [34]. For example, Rodriguez-Blanco et al. [31] developed a fault-detection technique of transistor short and open failures based on the transistor gate-voltage behavior to detect the overcurrent of the transistors. However, the technique needs a measurement system capable of detecting a delay time of $3 \mu \mathrm{s}$. Similarly, Lessmeier et al. [32] developed a diagnostic method to detect defects using two of the three phase current measurements of a synchronous motor. Bravo-Imaz et al. [8] used experimental data to detect three gear damage categories using total current signals. Dalla Vedova et al. [35] developed a fault detection algorithm for an aircraft electromechanical actuator (EMA) using a correlation coefficient calculated as a ratio between the actual EMA dynamic responses (motor torque and angular velocity) and the expected ones while using current as an input variable that impact the responses. Finally Ilonen et al., [9] used discriminative energy functions to analyze the frequency-domain regions of both vibration and stator current signals to identify induction motor bearing faults.

In both vibration and current base methods, signal filtering and processing techniques in conjunction with machine learning are needed for fault detection. Discrete wavelet transform [36] [29] [8], Fourier-Bessel expansion [6] [37] are examples of some of the time/frequency domain transformation techniques found in literature.

In all the above research contributions and regardless of the signal used, several challenges arose:

1. All model-based fault detection approaches require complex signal processing [8] and in some cases, the signal processing technique itself needs optimization.

2. Several features need to be estimated and monitored for fault detection simultaneously to overcome problems such as resolution and accuracy. Chandran et al. [38] proposed using 14 different features to diagnose gear faults such as root mean square (rms), average peak value, crest factor, etc. A subset of the features may be good enough to provide differentiation between healthy and faulty profiles [8]. Bellazzi and Jacazio et al., [37] pointed out that the implementation of accelerometric health monitoring of mechanical power drives has shown a considerable amount of false failure alarms and proposed the use of a combined application of several multivariate statistical techniques to improve the reliability of the diagnostic system.

3. Current and vibration measurement signatures are influenced by internal and external environment of the electromechanical system under assessment. Authors are not aware of any research except [37] where such environmental variables are used to calibrate the individual signature on each system measured.

4. Majority of research above is focused on isolation and detection of pure mechanical faults since electrical faults signatures are very fast and short. Very little research is done on detecting a mix bag of mechanical and electrical faults.

In this article, the intent is to isolate defects regardless of the source (mechanical or electrical) and without the need for complex signal processing or external measurements.

C. SPC Approach:

Statistical process control (SPC) is a well-known class of methods that has been extensively used in service and industry for quality improvement. In SPC, quality of a process or a product is assumed to be characterized by the distribution of a univariate characteristic or a multivariate distribution consists of several correlated characteristics or variables. Sometimes, one or more of these variables can be adequately explained as a function of one or more of the other variables, also referred to as profile or a signature [39]. Reis and Saraiva [40] provided a more general definition of profiles as: "data arrays, collected through automatic measurement systems, containing relevant information about how quality features are distributed in space and time that characterize a given product or process". This definition includes profiles used as inputs in process monitoring, whereas the output is the state of the process (healthy or faulty). Woodall et al. [41] provided an excellent review of the literature on SPC profile monitoring and discussed some of general issues with using profile SPC. These relationships or profiles can be linear [42] [43], or nonlinear [44] [45] [46] [47]. For example Kang and Albin [42] used linear profile monitoring to evaluate the process performance of a mass flow controller calibration in semiconductor manufacturing. Fan et al., 
[47] used the sum of sine functions as an SPC nonlinear profile to monitor the quality of the surface mount technology (SMT) reflow process. Jen and Fan [46] proposed change point analysis with second-order polynomial approximation between change point analysis to monitor the same reflow process originally introduced by Fan et al., [47]. Similarly, Amiri et al., [45] used a second-order polynomial profiles to monitor the quality of automotive engine components.

In profile monitoring, it is assumed that a random sample of size $n$ is collected for each $j^{\text {th }}$ profile over time of a response variable $y$ and explanatory variables $x$ 's. The observations can be summarized as $\left(x_{i p}, y_{i p}\right), i=1,2, \ldots n$, $j=1,2, \ldots, q$ where $x_{i j}$ represent an $i^{\text {th }}$ observation within a profile $j$. It is also assumed that a linear or non-linear model such as the two variables second-order model shown in equation (3) below can be adequately used for each $j^{\text {th }}$ profile.

$$
y_{j}=\beta_{0}+\beta_{1} x_{1}+\beta_{2} x_{2}+\beta_{12} x_{1} x_{2}+\beta_{11} x_{1}^{2}+\beta_{22} x_{2+}^{2} \varepsilon_{j}
$$

where $\beta^{\prime} s$ are model coefficients and $\varepsilon_{j}$ is a random variable representing model error.

Regardless of the shape of the profile, profile monitoring consists of two phases: phase I and phase II. In phase I, any outlying profiles are detected and assumed to be caused by a special assignable cause which needs to be removed. Once outliers are removed, an upper and lower control limits (UCL and LCL) are estimated and used in phase II. One of the most common statistics used in phase I is the Hotelling's $T^{2}$ statistic proposed by Brill [48] $T_{B, p}^{2}$ which is defined below:

$$
T_{B, p}^{2}=\left(\boldsymbol{b}_{p}-\overline{\boldsymbol{b}}\right)^{\prime} \boldsymbol{S}_{B}^{-1}\left(\boldsymbol{b}_{p}-\overline{\boldsymbol{b}}\right)
$$

where $\mathrm{b}$ is the vector of the least squares estimates of the coefficients, $\boldsymbol{b}=\left[\hat{\beta}_{0} \hat{\beta}_{1} \hat{\beta}_{2} \hat{\beta}_{12} \hat{\beta}_{11} \hat{\beta}_{22}\right]$ and $S_{B}$ is the sample variance-covariance matrix of $\boldsymbol{b}$ which can be estimated using the following:

$$
S_{B}^{2}\{b\}=\left[\begin{array}{cccccc}
S_{11}^{2} & S_{12} & S_{13} & S_{14} & S_{15} & S_{16} \\
S_{21} & S_{22}^{2} & S_{23} & S_{24} & S_{25} & S_{26} \\
& & & \cdot & & \\
& & & \cdot & & \\
S_{61} & S_{62} & S_{63} & S_{64} & S_{25} & S_{66}^{2}
\end{array}\right]
$$

The components $S_{11}^{2}, S_{22}^{2}, S_{33}^{2}, S_{44}^{2}, S_{55}^{2}, S_{66}^{2}$ are the estimated variances of $\hat{\beta}_{0}, \hat{\beta}_{1}, \hat{\beta}_{2}, \hat{\beta}_{12}, \hat{\beta}_{11}$, and $\hat{\beta}_{22}$ respectively. The $S_{12}, S_{13}, \ldots, S_{25}$ are the estimated covariance's between estimated coefficients. For example, $S_{12}$ is the covariance between $\hat{\beta}_{0}$ and $\hat{\beta}_{1}, S_{13}$ is the covariance between $\hat{\beta}_{0}$ and $\hat{\beta}_{2}$ and so on. In the case of having $q$ profiles and $k$ coefficients, Tracy et al., [49] proposed an approximation for $U C L$ for $T_{B, p}^{2}$ chart as shown below:

$$
U C L_{B}=\frac{(q-1)^{2}}{q} B_{1-\alpha, \frac{k}{2},(q-k-1) / 2}
$$

Where $\alpha$ represents the significance level which is assumed to be 0.05 in this article and $B$ is beta distribution with $k / 2$ and $(q-k-1) / 2$ degrees of freedom. Holmes and Mergen [50] pointed out that the previous method does not take into account the sequential sampling structure of the data and proposed another control charts based on the Hotelling's statistic shown in equation (7):

$$
T_{H, p}^{2}=\left(\boldsymbol{b}_{p}-\overline{\boldsymbol{b}}\right)^{\prime} \boldsymbol{S}_{H}^{-1}\left(\boldsymbol{b}_{p}-\overline{\boldsymbol{b}}\right)
$$

where the variance-covariance matrix $S_{H}$ is calculated based on successive difference of coefficient values from one profile to the other as shown below:

$$
\boldsymbol{S}_{H}=\frac{\widehat{\boldsymbol{V}}^{\prime} \widehat{\boldsymbol{V}}}{2(q-1)}
$$


where $\widehat{V}_{p}=\boldsymbol{b}_{p+1}-\boldsymbol{b}_{p}$ for $p=1, \ldots, q-1$ and $\boldsymbol{V}$ represents the matrix of $V_{p}$ estimates. Since $T_{H, p}^{2}$ relies on the successive difference in coefficients estimates, it is more robust than $T_{B, p}^{2}$ in detecting sustained shifts in the mean vector in phase I [51]. Scholz and Tosch [52] provided an approximation to the upper control limit of $T_{H, p}^{2}$ as shown below:

$$
\begin{aligned}
U C L_{H} & =\frac{(q-1)^{2}}{q} B_{1-\alpha, \frac{k}{2},(f-k-1) / 2} \\
\text { where } f & =\frac{2(q-1)^{2}}{3 q-4}
\end{aligned}
$$

Williams et al., [53] proposed an approximation of $T_{H, p}^{2}$ control chart for a large sample size where $q>k^{2}+3 k$ as shown below:

$$
U C L_{H}=\chi_{1-\alpha, k}^{2}
$$

It is generally believed that phase II is reasonably stable and any shifts happen are smaller in magnitude compared to phase I. As a result, more sensitive SPC charts are used such as cumulative sum (CUSUM) and exponentially weighted moving average (EWMA) where separate univariate chart is used for each and every coefficient.

In all cases surveyed in literature, the models used to describe profiles response are either use one or two explanatory variable [46] [45] [47] [54] or use a relatively simple model with few coefficients [55]. With the ever increase of industrial applications complexity, this might hinder the use of multivariate SPC for complex applications such as SFS under investigation. When the response model is oversimplified and only few linear combinations of the response are monitored and modeled, then some shifts in profiles are undetectable [53]. In this article, a simpler approach using defects chart (c-chart) where defects are defined as the outlying observations from healthy regression-based model is proposed and demonstrated using the SFS example.

\section{FUELS SYSTEM PROFILE MONITORING}

In complex systems in general, and SFS in specific, data is usually collected automatically using real time (on-line) measurement systems with high frequency sampling rates (above $1 \mathrm{KHz}$ ). As a result, fault detection techniques include a critical step where the size of the data collected is reduced (dimension reduction) by selecting only necessary data for fault detection. This step is called feature selection which will be discussed next for the SFS system under study followed by profile monitoring method techniques.

\section{A. Feature Selection of SFS}

Fifteen different variables were automatically recorded for each SFS system; among those variables are motor current $\left(I_{\text {motor }}\right)$ flow rate $(F R)$, duty cycle $(D C)$, fuel pressure $(P)$, and motor speed $(R P M)$. In order to ease calculations and processing time, a subset of this "big" data collected may be sufficient where few vital variables provide a good representation to the phenomenon under study. The selection of these vital variables, also called features, can be done through the understanding of the physics of the system, or using statistical tools such as correlation matrix or PCA, or a mix of both techniques. Figure 2 below depicts the correlation matrix between all the 15 variables of the SFS where the cell entries show the Pearson coefficient $(R)$ for each variable pair. Ten variables $\left(Z_{1}-Z_{10}\right)$ are strongly correlated to at least one of named five variables: $I_{\text {motor }}, F R, D C, P$, and $R P M$ shown in the last five columns with absolute value of $R$ of at least 0.75 . Both physics and correlation analysis supported the selection of the five primary features/variables above to isolate the excessive drawn current failure mode.

A PCA analysis was also done to all variables after normalization and led to the same conclusion that five variables can explain the majority of the variability, see Figure 3. Based on the \% Variation or eigenvalues, the proportion of variability explained by the last seven factors is minimal (less than 0.02 ) and they can be eliminated. The first five factors together represent $88 \%$ of the variability while seven factors explain $98.3 \%$ of the variability.

\begin{tabular}{|c|c|c|c|c|c|c|c|c|c|c|c|c|c|c|c|}
\cline { 2 - 14 } \multicolumn{1}{c|}{} & $\mathrm{Z}_{1}$ & $\mathrm{Z}_{2}$ & $\mathrm{Z}_{3}$ & $\mathrm{Z}_{4}$ & $\mathrm{Z}_{5}$ & $\mathrm{Z}_{6}$ & $\mathrm{Z}_{7}$ & $\mathrm{Z}_{8}$ & $\mathrm{Z}_{9}$ & $\mathrm{Z}_{10}$ & $\mathrm{FR}$ & $\mathrm{DC}$ & $\mathrm{RPM}$ & $\mathrm{I}$ & $\mathrm{P}$ \\
\hline $\mathrm{Z}_{1}$ & & 0.06 & -0.06 & 0.04 & 0.77 & 0.47 & 0.95 & -0.11 & 0.04 & -0.24 & -0.20 & 0.82 & 0.06 & 0.78 & 0.04 \\
\hline
\end{tabular}




\begin{tabular}{|c|}
\hline$Z_{2}$ \\
\hline$Z_{3}$
\end{tabular}

Figure 2 Correlation map with value of $\mathrm{R}$ for each variable pair

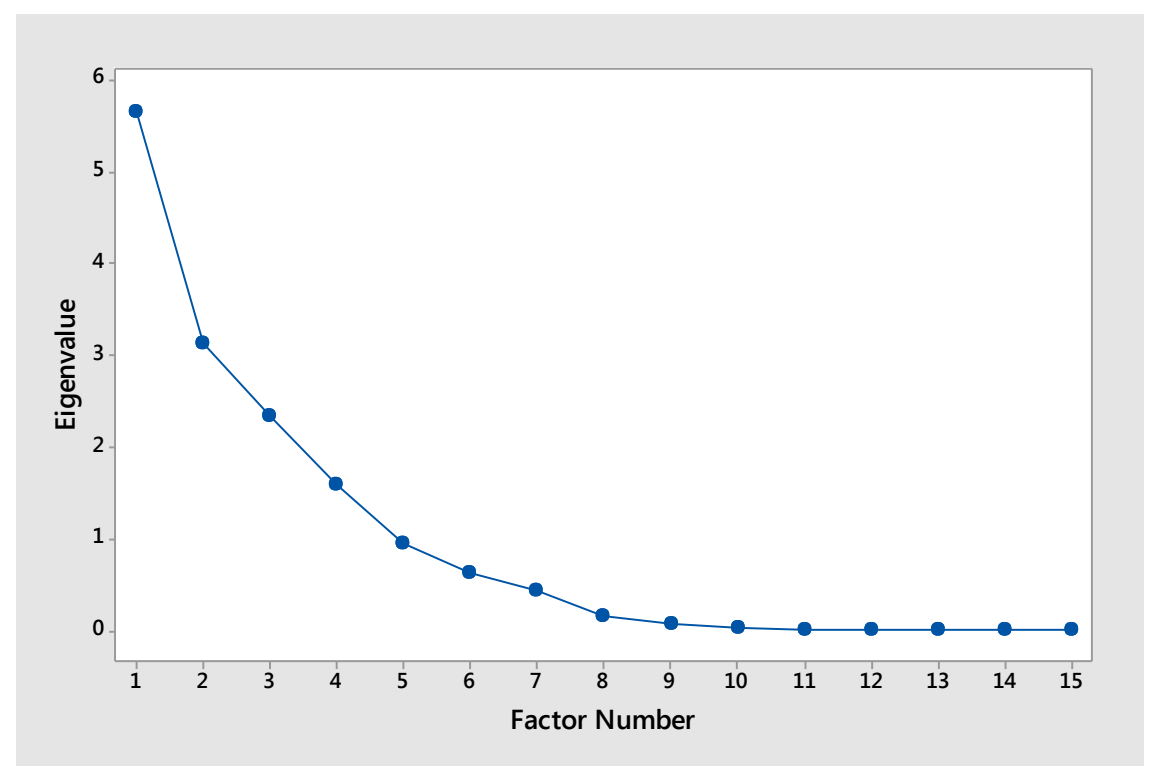

Figure 3 PCA Scree plot for the SFS

\section{B. Profile Monitoring for Fault Detection}

In this section, the control charts based on the Hotelling's $T^{2}$ statistic will be used first to monitor the quality of SFS systems. The applicability of the Hotelling method and some implementation issues will be addressed by introducing a new method based on defects monitoring control charts.

In this study, a set of 14 profile data will be used for phase I in which the SFS systems were released to field and proven to be functioning properly, i.e. healthy. An additional six profiles with three proven healthy ones and three faulty ones will be used to validate the accuracy of the SPC method used in phase II. Each profile consists of at least 13600 measurement observations of the five parameters/features selected. A sample of data collected is shown in Appendix I. The motor drawn current is considered as a response variable and $F R, D C, R P M$, and $P$ are the explanatory variables. The SFS system is composed of a large number of components and subsystems with inherent variability of each one of these components. As a result, this component variability is expected to propagate into the SFS observed motor current accordingly and can be seen when different healthy profiles are compared. Figure 4 
depicts some of the raw data of some of healthy and failed profiles. It is evident that profile-to-profile variability is present, for example there is a 1.5A difference between the first $(\mathrm{P} 1-\mathrm{H})$ and fourth profile $(\mathrm{P} 4-\mathrm{H})$ and both profiles are performing well (healthy) in the field. The current of the motor depends on several variables such as the load exerted on the pump, the efficiency of the pump especially the drag force created by the friction of the bearings in the pump, the efficiency of the motor itself, the performance of the IGBT transistors under high temperature, and finally the quality of the power supply.

In addition to SFS-to-SFS hardware variability, usage of these systems in the field is different from one location to the other due to differences in external environment, duty cycle, and user preference. Some clients run the SFS using a constant load mode, others run it using constant speed mode depending on application. Moreover, each client has a preference in terms of flow rate and pressure which are driven by the speed and motor torque which are controlled by client. As a result, the testing profile of the EOL quality test is slightly different from one SFS to the other in terms of ranges of input variables and number of observations depending on client requests. This variability add complexity to the anomaly detection problem on hand since majority of SPC methods surveyed assumes identical measurement points within each profile. To overcome such a challenge, a common measurement region in $\mathrm{X}$ space between all different modes of testing is selected and used for profile comparisons. The selected region covers a typical range of normal operating conditions where the range of each variable $x_{i}$ is the same for all profiles. Although ranges are the same for all input variables, number of observations within each profile is different. However, number of observations in each profile is large enough to guarantee a balanced coverage of measurement region and provide accurate prediction models to be used later on.

For each SFS in the first 14 SFS healthy systems, observation data was used to build a regression model which is simple and can explain a significant percentage of variability in the data. The latter condition can be validated using the adjusted coefficient of determination $R_{a d j}^{2}$ which takes into consideration the number of terms used in the model. Different regression models were tried to fit the data on hand and the quadratic polynomial model shown in equation (11) showed a good fit with minimum number of terms.

$$
\begin{gathered}
y_{j}=\beta_{0}+\beta_{1} X_{1}+\beta_{2} X_{2}+\beta_{3} X_{3}+\beta_{4} X_{4}+\beta_{11} X_{1}^{2}+\beta_{22} X_{2}^{2}+\beta_{44} X_{4}^{2}+ \\
\beta_{13} X_{1} X_{3}+\beta_{14} X_{1} X_{4}+\beta_{23} X_{2} X_{3}+\beta_{34} X_{3} X_{4}+\varepsilon_{j}
\end{gathered}
$$

where $y_{j}$ is the vector of motor current for SFS $j$ (profile $j$ ), $X_{1}, X_{2}, X_{3}$ and $X_{4}$ are the vectors of $F R, D C, R P M$, and $P$ respectively, $\varepsilon_{j}$ is the error vector. The parameters $\beta_{0}-\beta_{34}$ are model coefficients which are estimated for each profile using the least square method based on error minimization. It is worth mentioning here that the original values of explanatory variables were scaled down to $[-1,1]$ range so that the estimates are of similar magnitude and the coefficient estimates can be compared as an indication of the effect of the explanatory variable. The estimates of the regression parameters and $\mathrm{p}$-values of their significance along with the model coefficient of determination $\left(R_{a d j}^{2}\right)$ are shown in Table 1. The first entry in each cell of Table 1 is the estimated coefficient while the second entry shown in parenthesis is the p-value. The adjusted coefficient of determination $R_{a d j}^{2}$ ranges from 92 to $99 \%$ which confirms that the model fits data collected very well. Analysis of variance (ANOVA) and residual analysis were conducted as well, but not shown here, to confirm the significance of the regression parameters and the normality of the error term $\varepsilon_{j}$ with mean zero and constant variance respectively. 


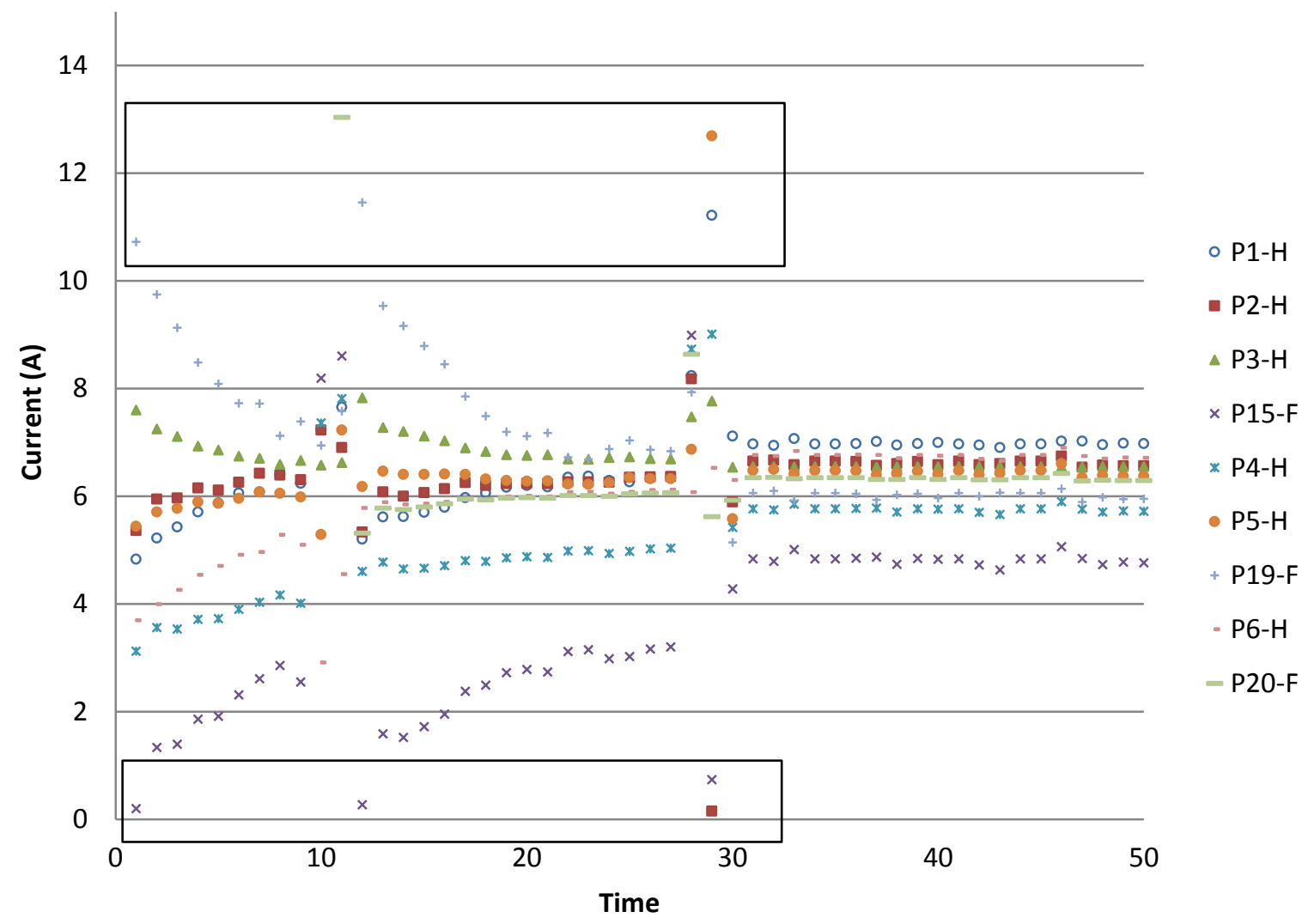

Figure 4 Sample of profiles raw data

Table 1 SFS profile modeling results for Phase I

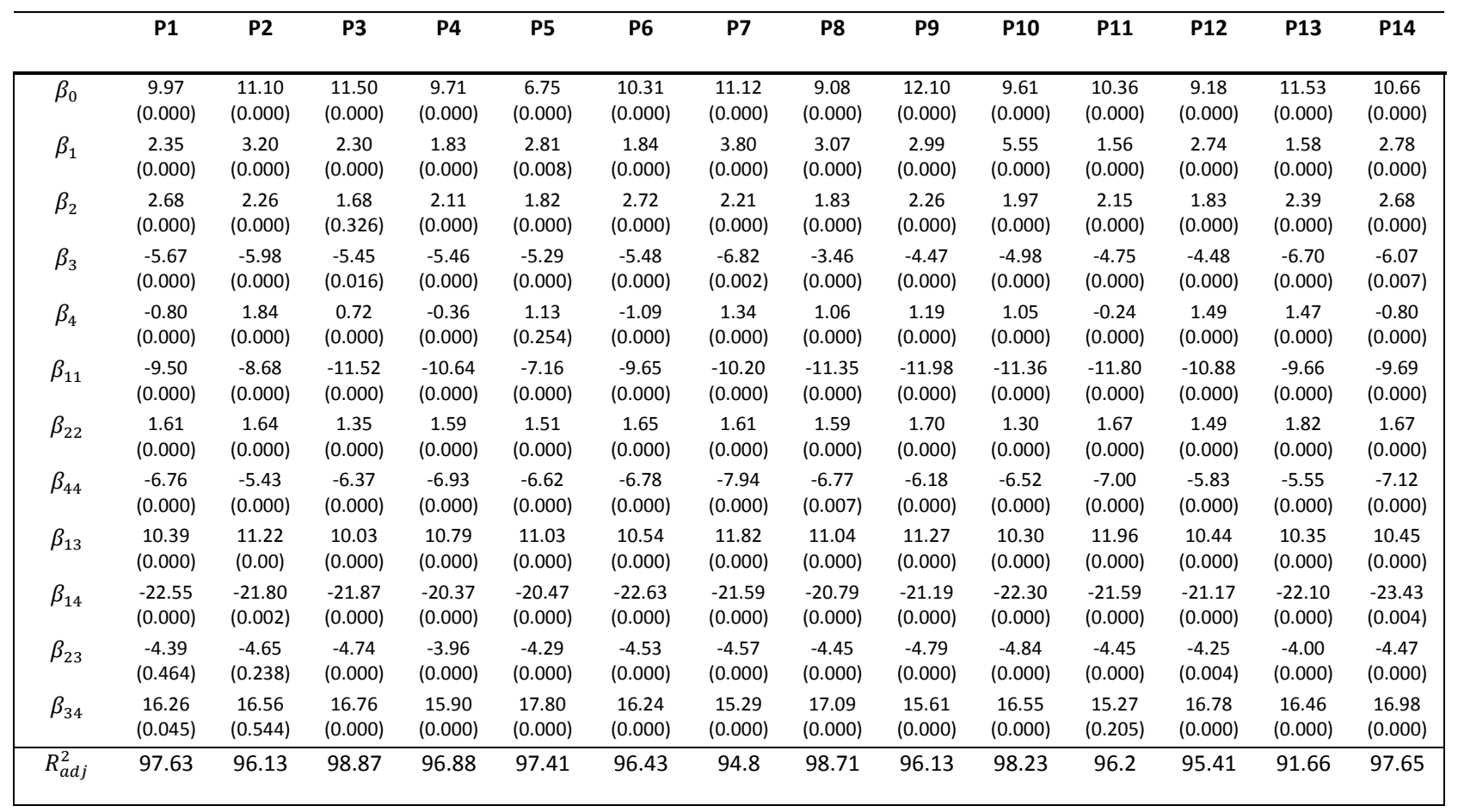


Next the variance-covariance matrix $S_{B}$ were calculated as proposed by [48] and shown in Appendix II. For phase I, the $T_{B, p}^{2}$ is estimated for every profile and depicted in Figure 5. The UCL is calculated as indicated by equation (6) above and using significance level $\alpha$ of 0.05 as follows:

$$
U C L_{B}=\frac{(14-1)^{2}}{14} B_{0.05,6,0.5}=12.07
$$

The $T_{B}^{2}$ control chart show that all phase I profiles, which are known to be healthy beforehand, are below the UCL and process is stable. Similarly, the same quadratic model was used to fit the data of each profile in phase II. Table 2 summarizes the results of the fitted models of phase II profiles. The $T_{B, p}^{2}$ estimates for phase II profile are also calculated and plotted on Figure 5 as well. All known failed profiles of phase II (P15, P19, and P20) had a $T_{B}^{2}>$ $U C L$ and the chart truly classified the process generated these profiles as "out of control". However, two of the known healthy profiles of phase II, namely P16 and P18, were mistakenly classified as "out of control" as well. This is due to the high number of model parameters where a slight shift in majority of model coefficients may result in a high Hotelling's $\mathrm{T}^{2}$ statistic and cause a false rejection alarm. To demonstrate this point, let's compare the parameter deviation from average vector $\left(\boldsymbol{b}_{p}-\overline{\boldsymbol{b}}\right)^{\prime}$ for profile 17 (true healthy) and profile 18 (false alarm):

$$
\begin{aligned}
\left(\boldsymbol{b}_{17}-\overline{\boldsymbol{b}}\right)^{\prime} & =\left[\begin{array}{lllll}
-0.03-0.04-0.07-0.13-0.02-1.54 & 0.00-0.49-0.09-0.03 & 0.28-0.46
\end{array}\right] \\
\left(\boldsymbol{b}_{18}-\overline{\boldsymbol{b}}\right)^{\prime} & =\left[\begin{array}{lllll}
-0.11-0.14 & 0.31 & 0.33 & 0.03-0.23-0.03-0.27-0.24-0.27 & 0.12-0.10
\end{array}\right]
\end{aligned}
$$

Profile 17 has high absolute deviations in three elements, namely sixth, eighth, and twelve while all deviations in profile 18 is lower in magnitude but more frequent. In other words, profile 18 exhibits more deviation across all parameters with lower magnitude. Therefore, the more terms the regression model has the more sensitive the Hotelling's $T^{2}$ statistic to slighter changes in the regression coefficient estimates.

In the case of $T_{H}^{2}$ chart, the combination of model terms $k=12$ and number of profiles $q=14$ do not support the

\begin{tabular}{|c|c|c|c|c|c|c|}
\hline & P15-F & P16-H & P17-H & P18-H & P19-F & P20-F \\
\hline$\beta_{0}$ & $\begin{array}{c}10.20 \\
(0.000) \\
\end{array}$ & $\begin{array}{c}11.64 \\
(0.000) \\
\end{array}$ & $\begin{array}{c}10.18 \\
(0.000) \\
\end{array}$ & $\begin{array}{c}10.10 \\
(0.000)\end{array}$ & $\begin{array}{c}9.15 \\
(0.000)\end{array}$ & $\begin{array}{c}10.24 \\
(0.000) \\
\end{array}$ \\
\hline$\beta_{1}$ & $\begin{array}{c}3.00 \\
(0.000)\end{array}$ & $\begin{array}{c}2.41 \\
(0.000)\end{array}$ & $\begin{array}{c}2.70 \\
(0.000)\end{array}$ & $\begin{array}{c}2.60 \\
(0.000)\end{array}$ & $\begin{array}{c}1.48 \\
(0.000)\end{array}$ & $\begin{array}{c}1.81 \\
(0.000)\end{array}$ \\
\hline$\beta_{2}$ & $\begin{array}{c}2.48 \\
(0.000) \\
\end{array}$ & $\begin{array}{c}2.03 \\
(0.000) \\
\end{array}$ & $\begin{array}{c}2.11 \\
(0.000)\end{array}$ & $\begin{array}{c}2.49 \\
(0.000)\end{array}$ & $\begin{array}{c}2.05 \\
(0.000)\end{array}$ & $\begin{array}{c}2.33 \\
(0.000)\end{array}$ \\
\hline$\beta_{3}$ & $\begin{array}{c}-6.83 \\
(0.007)\end{array}$ & $\begin{array}{c}-6.57 \\
(0.000)\end{array}$ & $\begin{array}{c}-5.49 \\
(0.000)\end{array}$ & $\begin{array}{c}-5.03 \\
(0.000)\end{array}$ & $\begin{array}{c}-4.52 \\
(0.000)\end{array}$ & $\begin{array}{c}-5.03 \\
(0.000)\end{array}$ \\
\hline$\beta_{4}$ & $\begin{array}{c}0.70 \\
(0.000) \\
\end{array}$ & $\begin{array}{c}1.68 \\
(0.000) \\
\end{array}$ & $\begin{array}{c}0.55 \\
(0.000) \\
\end{array}$ & $\begin{array}{c}0.60 \\
(0.000)\end{array}$ & $\begin{array}{c}-0.22 \\
(0.000)\end{array}$ & $\begin{array}{c}0.68 \\
(0.000) \\
\end{array}$ \\
\hline$\beta_{11}$ & $\begin{array}{l}-11.00 \\
(0.000)\end{array}$ & $\begin{array}{c}-9.20 \\
(0.000)\end{array}$ & $\begin{array}{l}-11.83 \\
(0.000)\end{array}$ & $\begin{array}{c}-10.52 \\
(0.000)\end{array}$ & $\begin{array}{c}-11.21 \\
(0.000)\end{array}$ & $\begin{array}{l}-10.48 \\
(0.000)\end{array}$ \\
\hline$\beta_{22}$ & $\begin{array}{c}1.40 \\
(0.000)\end{array}$ & $\begin{array}{c}1.33 \\
(0.000)\end{array}$ & $\begin{array}{c}1.59 \\
(0.000)\end{array}$ & $\begin{array}{c}1.56 \\
(0.000)\end{array}$ & $\begin{array}{c}1.50 \\
(0.000)\end{array}$ & $\begin{array}{c}1.58 \\
(0.000)\end{array}$ \\
\hline$\beta_{44}$ & $\begin{array}{c}-7.94 \\
(0.000)\end{array}$ & $\begin{array}{c}-6.36 \\
(0.000)\end{array}$ & $\begin{array}{c}-7.05 \\
(0.000)\end{array}$ & $\begin{array}{c}-6.83 \\
(0.000)\end{array}$ & $\begin{array}{c}-6.65 \\
(0.003)\end{array}$ & $\begin{array}{c}-6.84 \\
(0.000)\end{array}$ \\
\hline$\beta_{13}$ & $\begin{array}{c}10.20 \\
(0.000)\end{array}$ & $\begin{array}{c}10.33 \\
(0.000) \\
\end{array}$ & $\begin{array}{c}10.74 \\
(0.000) \\
\end{array}$ & $\begin{array}{c}10.59 \\
(0.000)\end{array}$ & $\begin{array}{c}11.36 \\
(0.000)\end{array}$ & $\begin{array}{c}10.62 \\
(0.000)\end{array}$ \\
\hline$\beta_{14}$ & $\begin{array}{c}-17.82 \\
(0.000)\end{array}$ & $\begin{array}{l}-17.49 \\
(0.000) \\
\end{array}$ & $\begin{array}{l}-21.73 \\
(0.000)\end{array}$ & $\begin{array}{c}-21.97 \\
(0.000)\end{array}$ & $\begin{array}{c}-20.51 \\
(0.000)\end{array}$ & $\begin{array}{l}-21.59 \\
(0.000)\end{array}$ \\
\hline$\beta_{23}$ & $\begin{array}{c}-4.31 \\
(0.000)\end{array}$ & $\begin{array}{c}-3.90 \\
(0.000)\end{array}$ & $\begin{array}{c}-4.18 \\
(0.000)\end{array}$ & $\begin{array}{c}-4.34 \\
(0.000)\end{array}$ & $\begin{array}{c}-4.23 \\
(0.000)\end{array}$ & $\begin{array}{c}-4.40 \\
(0.000)\end{array}$ \\
\hline$\beta_{34}$ & $\begin{array}{c}13.18 \\
(0.000) \\
\end{array}$ & $\begin{array}{c}12.62 \\
(0.000) \\
\end{array}$ & $\begin{array}{c}15.94 \\
(0.000) \\
\end{array}$ & $\begin{array}{c}16.30 \\
(0.006)\end{array}$ & $\begin{array}{c}15.50 \\
(0.000)\end{array}$ & $\begin{array}{c}16.32 \\
(0.000) \\
\end{array}$ \\
\hline$R_{a d j}^{2}$ & 95.1 & 92.97 & 97.97 & 95.69 & 91.12 & 91.88 \\
\hline$T_{B, j}^{2}$ & 58.00 & 35.94 & 6.36 & 13.18 & 89.13 & 16.06 \\
\hline
\end{tabular}
assumption of $q>k^{2}+3 k$ which is necessary to $U C L_{H}$ approximation shown in equation (10).

Table 2 Phase II modeling summary 


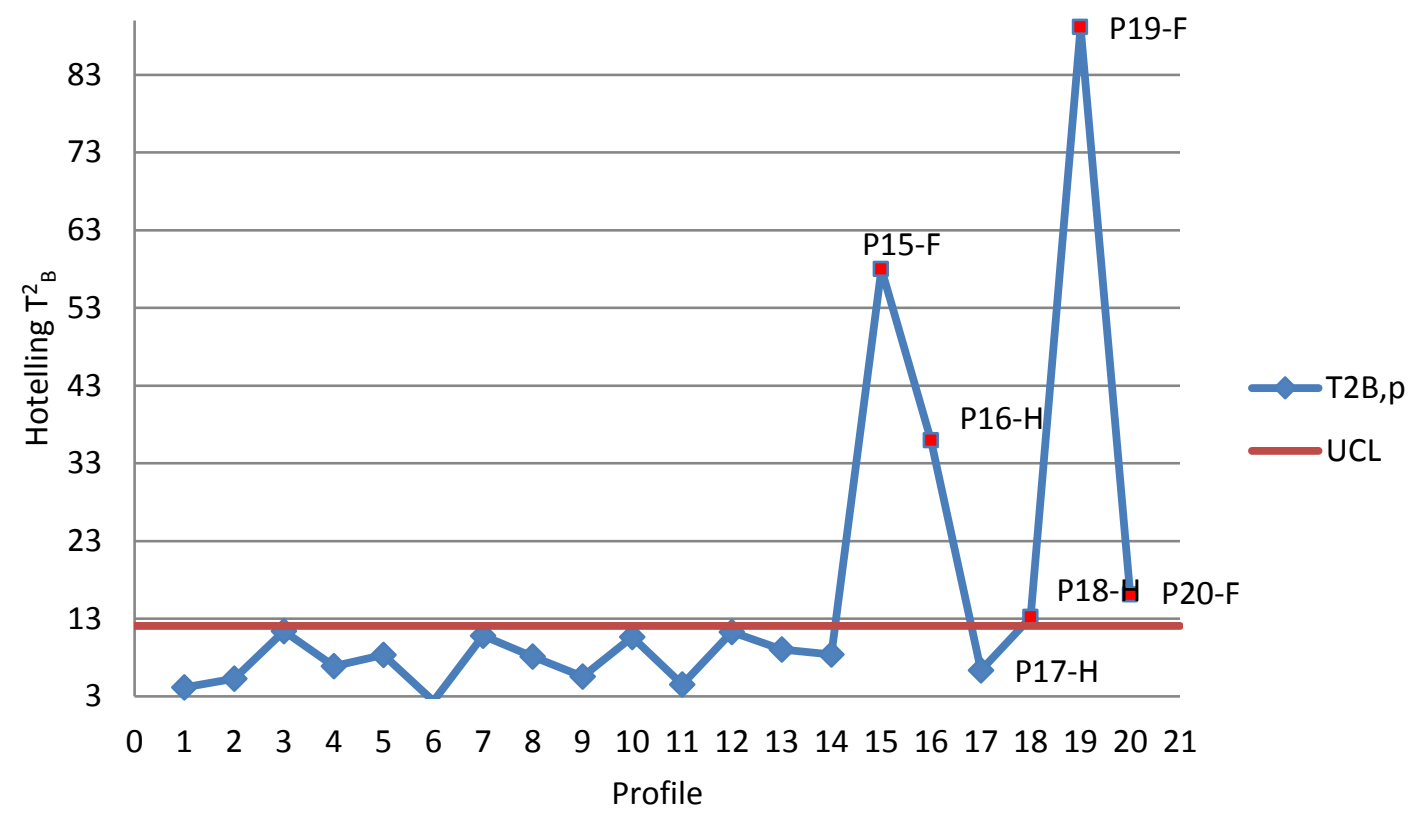

Figure $5 \mathrm{~T}^{2}{ }_{\mathrm{B}, \mathrm{P}}$ control chart based on Brill estimate

The SFS profile parametric fit is complex and has too many parameters which makes the use of traditional control charts based on the Hotelling's $T^{2}$ statistic challenging and vulnerable to false alarms as shown above. As an alternative approach, a healthy "baseline" region is constructed based on phase I healthy profiles and used to compare all other profiles. Once the baseline region is determined, an "outlying observations" measure from this region is used to identify the count of observations outside this region and use it to identify faulty profiles. This approach is similar in principle to the comparison of the fit of a profile with the average fit of healthy profiles and uses the difference to detect faulty behaviors. The region comparison is more powerful since the healthy profileprofile variability is considered in the determination of unusual observations. The details of the proposed approach are shown below:

1. Based on the common measurement space observations, develop a non-linear regression model for each profile in phase I, i.e. training set as shown in equation 11 and Table 1.

2. Generate random values of input variables within ranges specified by the selected common region in $\mathrm{X}$ space and governed by the best distribution that fit a sample of data collected from the field for each input variable. Since the random values of each input variable will be generated independently from other input variables, it is expected that some combinations of input variables will not be feasible, i.e. combination cannot happen in real life operation and should be deleted. In the SFS case study, 13,000 random numbers were generated using best-fit distributions summarized in Table 3 below. The resultant 13,000 rows by four columns matrix will be referred to as testing matrix. Having a unique testing matrix is necessary for the use of nonconforming count control chart (c-chart) which will be discussed in step 5. The c-chart requires a constant number of observations in each sample/profile which is achieved by using the training matrix.

Table 3 SFS input variables distributions

\begin{tabular}{l|ll}
\hline Input variable & Best-fit distribution & Parameters \\
\hline Flow rate & Uniform & $\begin{array}{l}\text { Minimum value }=-1 \\
\text { Maximum value }=1\end{array}$ \\
\hline Duty cycle & Uniform & $\begin{array}{l}\text { Minimum value }=-1 \\
\text { Maximum value }=1\end{array}$ \\
& & Mean $=-0.14519$ \\
RPM & Normal & Variance $=0.07131$ \\
\hline Pressure & & Mean $=0.355$ \\
& Normal & Variance $=0.027$ \\
\hline
\end{tabular}


3. Use regression models developed in step 1 to predict motor current values for the testing matrix for each of phase I regression models.

4. Using phase I profiles, i.e. known healthy profiles, determine if predicted values of motor current has any outlying observations using Grubbs' test [56]. Although outliers, in the general sense, are symptoms of errors in the data collection and statistical model fitting, it is used in this article as a deviation of the predicted output in phase II from actual known healthy profiles. Grubbs test depends on whether you test the smallest value, $y_{i}$ or the largest value, $y_{n}$. For example to test whether $y_{n}$, is an outlier, then Grubbs' test statistic G can be written as:

$$
G_{u}=\frac{y_{n}-\bar{y}}{s}
$$

Similarly, to test whether $y_{i}$, is an outlier, then Grubbs' test statistic can be written as:

$$
G_{L}=\frac{\bar{y}-y_{i}}{s}
$$

where $\bar{y}$ and $s$ are the sample mean and standard deviation respectively, $y_{i}$ is the smallest value in the sample and $n$ is the number of observations in the sample. $G$ is a Grubbs' test statistic; a random variable follow a t-distribution with $n-2$ degrees of freedom and the $p$-value can be approximated as [56]:

$$
p \approx n \operatorname{Prob}\left(t>\sqrt{\frac{n(n-2) G^{2}}{(n-1)^{2}-n G^{2}}}\right)
$$

It is worth noting that if a sample contains more than one suspected outlier, then Grubbs' test ratio may not be effective. However, since each point for each profile will be tested individually Grubbs test is accurate enough.

5. Count number of outlying observations for each profile by evaluating each current point in phase I and develop a c-chart for outlying observations where the control limits are based on total number of outlying observations per profile:

$$
c_{i}=\sum_{k=0}^{13,000} O_{i}
$$

where $O_{i}$ is number of outlying observations for profile $p$ over the 13,000 testing matrix points. The upper and lower control limits, $U C L$ and $L C L$ respectively, can be estimated using phase I profiles as shown below:

$$
\begin{aligned}
U C L & =\bar{c}+3 \sqrt{\bar{c}} \\
L C L & =\bar{c}-3 \sqrt{\bar{c}}
\end{aligned}
$$

Two important points has to be discussed here, first regarding the definition of outlier, and secondly regarding the use of c-charts. Although outlying observations are used to detect any errors of data collected or measurements, we use the terminology of outlying observations here as any deviation of the individual predicted profile from the known healthy profiles data set. This deviation is not due to data collection error, it is rather due to faulty behavior of the SFS system in certain areas of measurement space. Secondly, the use of c-charts is typically used for count of nonconforming or defects, in our proposed method the deviation from healthy performance is considered to be a defect.

6. Once process is stable and all "out of control" profiles are eliminated in phase I, phase II profiles can be modeled and outlying observations are counted as shown in steps 4 and 5. The two rectangles in Figure 4 enclose some of the outlying observations for some of the profiles. 
Figure 6 below summarizes the results of c-chart for the SFS systems. All three failed profiles had number of outlying observations beyond UCL and were designated as out of control. Healthy profiles were within limits and were designated as such.

The above proposed method is very similar in nature to the Hotelling method in principle. The hotelling $T^{2}$ is based on deviations of points $(x-\mu)$ vector or coefficients of regression models $(b-\bar{b})$ from mean vector while the outlying observations are identified based on deviations from sample means.

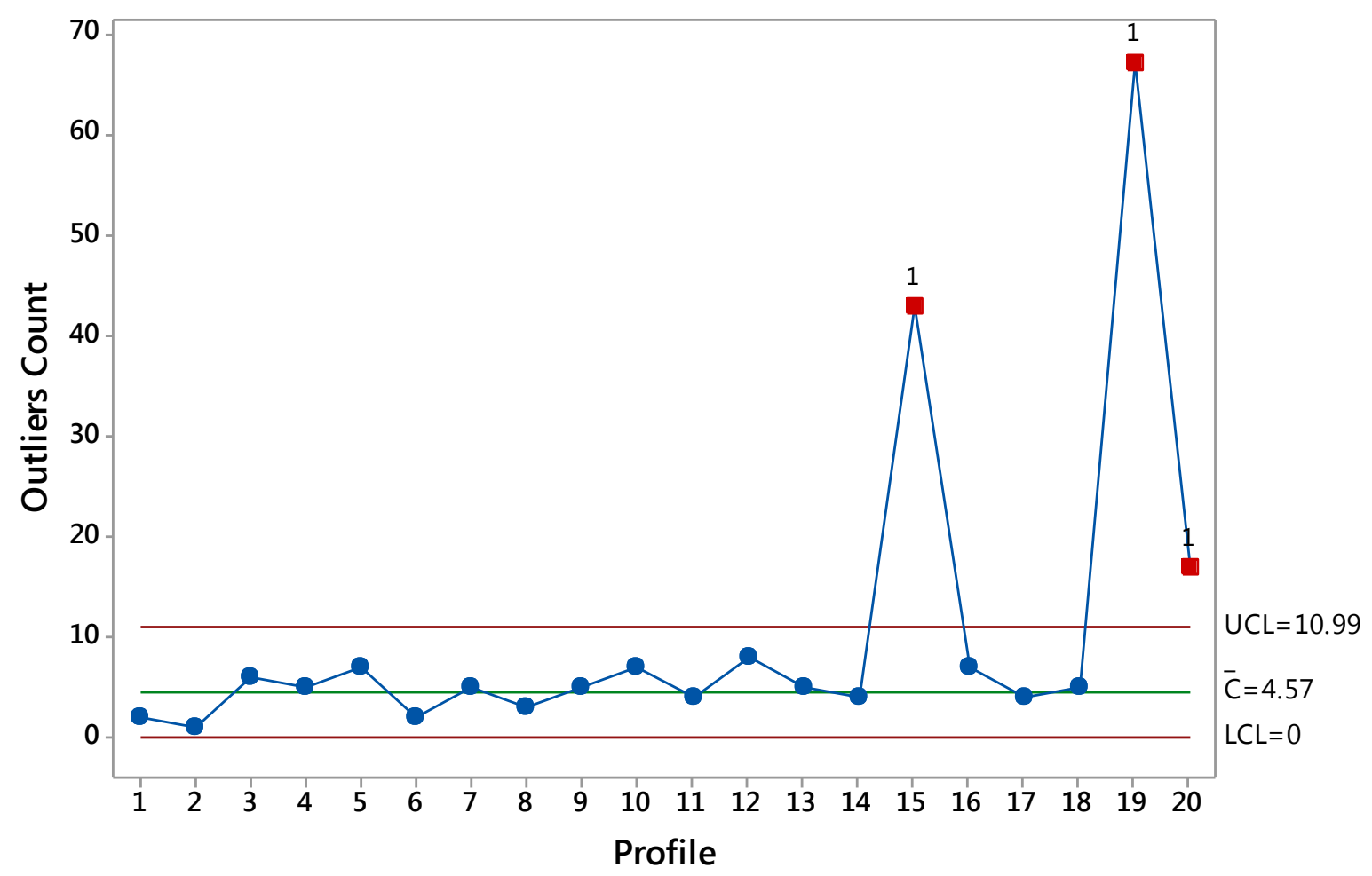

Figure 6 c-chart for SFS using proposed method

In the c-chart, each measurement within the profile is treated as an area of opportunity for failure and since there is more than 13,000 points in each profile, the usage of $\mathrm{c}$-chart was appropriate. The lower the number of measurement points, the lower the chances of detecting defects and less useful the c-chart will be. Another enabler for the proposed method is the magnitude and significance of profile-to profile variability which can be mistakenly ignored and treated as a special cause. Williams [53] pointed out that profile-to-profile common cause variations is a basic issue in all applications since the variation between profiles should be incorporated into control charts limits. Interrogating the individual measurement points is more effective in capturing profile-to-profile variability than model parameter coefficients since the latter is based on finding the best fit curve. It is worth mentioning that the proposed approach is similar in nature to the analytical redundancy fault identification methods which have been utilized in the control of safety critical systems control [11]. In analytical redundancy, a critical measurement is compared to another redundant measurement obtained from the functional dependence among the process variables and is usually provided by a set of algebraic or temporal relationships among the inputs and outputs of the system. Any inconsistency is considered as a potential fault of the system. In all cases surveyed in literature, the models used to describe the response profiles are either use one or two explanatory variable [46] [45] [47] [54] or uses a relatively simple model with few coefficients [55]. With the ever increase of industrial applications complexity, this might hinder the use of multivariate SPC for complex applications such as SFS under investigation. When the response model is oversimplified and only few linear combinations of the response are monitored and modeled, then some shifts in profiles are undetectable [53]. 


\section{CONCLUSIONS}

A data driven methodology using outlying observations detection technique and c-chart is proposed to detect smart fuel system anomalies. The methodology use prediction polynomial regression models of output variable (current) as a function of four input variables. The anomalies have one symptom, excessive motor current drawn, with different electrical and mechanical causes. Traditional control charts based on the Hotelling's $T^{2}$ statistic failed short in terms of detecting true positive, i.e. healthy profiles due to profile-to-profile variability and sensitivity of these methods to the complexity of non-linear models used. Moreover, the majority of existing parametric model methods is based on simultaneous monitoring of each parameter with separate chart unless estimators are dependent. This approach is valid when number of parameters is small, otherwise the significance of the test will be more challenging to meet.

The methodology presented in this paper is scalable and can be applied to a wide range of systems to assess their health from inspection check to anticipate and avoid failures. This will result in an increased operational availability and reduced costs to the clients.

\section{REFERENCES}

[1] N. Vichare and M. Pecht, "Prognostics and health management of electronics," IEEE Transactions on Components and Packaging Technologies, vol. 29, no. 1, 2006.

[2] B. Ferrell, , "JSF Prognostics and Health Management," in IEEE Aerospace Conference, 1999.

[3] M. Pepe, The Statistical Evaluation of Medical Tests for Classification and Prediction, Oxford, UK: Oxford University Press, 2003.

[4] O. Gusikhin, N. Rychtyckyj and D. Filev, "Intelligent Systems in the Automotive Industry: Applications and Trends," Knowledge and Information Systems, vol. 12, pp. 147-168, 2007.

[5] A. Saxena, J. Celaya, E. Balaban, B. Sabha and K. Goebel, "Metrics for Evaluating Performance of Prognostic Techniques," in International Conference on Prognostics and Health Management Proceedings, 2008.

[6] V. Tran, F. Althobiani, A. Ball and B.-K. Choi, "An application to transient current signal based induction motor fault diagnosis of Fourier-Bessel expansion and simplified fuzzy ARTMAP," Expert Systems with Applications, vol. 40, no. 13, pp. 5372-5384, 2013.

[7] C. Kar and A. Mohanty, "Monitoring gear vibrations through motor current signature analysis and wavelet transform," Mechanical Systems and Signal Processing, vol. 47, no. 5, pp. 199-221, 2006.

[8] I. Bravo-Imaz, A. Garcia-Arribas, S. Ferreriro, S. Fernandez and A. Arnaiz, "Motor current signature analysis for gearbox health monitoring: Experiment, signal analysis and classification," in European Conference of the Prognostics and Health Management Society 2014, Nantes, France, 2014.

[9] J. Ilonen, J.-K. Kamarainen, T. Lindh, J. K. H. Ahola and J. Partanen, "Diagnosis tool for motor condition monitoring," IEEE transactions on Industry Applications, vol. 41, no. 4, pp. 963-972, 2005.

[10] A. Das, J. Maiti and R. Banerjee, "Process monitoring and fault detection strategies: a review," International Journal of Quality \& Reliability Management, vol. 29, no. 7, pp. 720-752, 2012.

[11] V. Venkatasubramanian, R. Yin and S. Kavuri, "A review of process fault detection and diagnosis. Part I - III," Computers and Chemical Engineering, vol. 27, pp. Part I: 293-311, Part II: 313-326, Part III: 327-346, 2003.

[12] Z. Ge, Z. Song and F. Gao, "Review of recent research on data-based process monitoring," Industrial \& Engineering Chemistry Research, vol. 52, pp. 3543-3562, 2013.

[13] K. Severson, P. Chaiwatanodom and R. Braatz, "Perspective on Process Monitoring of Industrial Systems," Proceedings of the 9th IFAC Symposium on Fault Detection, Supervision, and Safety for Technical Processes, Paper FrAP6.1., pp. 931-939, 2015.

[14] T. Rato and M. Reis, " Fault detection in the Tennessee Eastman process using dynamic principal components analysis with decorrelated residuals (DPCA-DR)," Chemometrics and Intelligent Laboratory Systems, vol. 125, pp. 101-108, 2013.

[15] P. Nomikos and J. F. MacGregor, "Multivariate SPC charts for monitoring batch processes," Technometrics, vol. 37, no. 1, pp. 41-59, 1995.

[16] T. Rato and M. S. Reis, "Advantage of Using Decorrelated Residuals in Dynamic Principal Component 
Analysis for Monitoring Large-Scale Systems," Industrial \& Engineering Chemistry Research, vol. 52, no. 38, pp. 13685-13698, 2013.

[17] T. Rato and M. Reis, " Defining the structure of DPCA models and its impact on process monitoring and prediction activities," Chemometrics and Intelligent Laboratory Systems , vol. 125, pp. 74-86, 2013.

[18] E. Russell, L. Chiang and R. Braatz, "Fault detection in industrial processes using canonical variate analysis and dynamic principal component analysis," Chemometrics and Intelligent Laboratory Systems, Vols. 81-93, p. $51,2000$.

[19] T. Komulainen, M. Sourander and S.-L. Jamsa-Jounela, "An online application of dynamic PLS to a Dearrmatization process," Comput. Chem. Eng., vol. 28, pp. 2611-2619, 2004.

[20] B. Bakshi, "Multiscale PCA with Application to Multivariate Statistical Process Control.," AIChE Journal, vol. 44, no. 7, pp. 1596-1610, 1998.

[21] M. Reis, B. Bakshi and P. Saraiva, "Multiscale statistical process control using wavelet packets," AIChE Journal, vol. 54, no. 9, pp. 2366-2378, 2008.

[22] M. Reis and P. Saraiva, " Multiscale Statistical Process Control with Multiresolution Data.," AIChE Journal, vol. 52, no. 6, pp. 2107-2119, 2006.

[23] M. Reis, "An integrated multiscale and multivariate image analysis framework for process monitoring of colour random textures: MSMIA," Chemometrics and Intelligent Laboratory Systems, vol. 142, pp. 36-48, 2015.

[24] T. Rato and M. Reis, "Sensitivity enhancing transformations for monitoring the process correlation structure," Journal of Process Control, vol. 24, pp. 905-915, 2014.

[25] W. Ku, R. Storer and C. Georgakis, "Disturbance detection and isolation by dynamic prinicpal component analysis," Chemometrics and Intelligent Labratory Systems, vol. 30, pp. 179-196, 1995.

[26] L. Chiang and R. Braatz, " Process Monitoring using the causal map and multivariate statistics: fault detection and identification," Chemometrics and Intelligent Laboratory Systems, vol. 65, pp. 159-178, 2003.

[27] M. Reis and P. Saraiva, " Heteroscedastic latent variable modelling with applications to multivariate statistical process control," Chemometrics and Intelligent Laboratory Systems, vol. 80, pp. 57-66, 2006.

[28] J. R. Stack, T. G. Habelter and R. G. Harley, "Fault classification and fault signature production for rolling element bearings in electric machines," in IEEE International Symposium on Diagnostics for Electric Machines, Power Electronics and Drives, Atlanat, GA, USA, 2004.

[29] J. K. Kimotho and W. Sextro, "An approach for feature extraction and selection from non-trending data for machinery prognosis," in European Conference of prognostics and health Management Society, Nantes, France, 2014.

[30] J. Tian, M. H. Azarian and M. Pecht, "Anamoly detection using self-organizing maps-based K-nearest nieghbor algorithms," in European Conference of the Prognostics and Health Management Society 2014, Nantes, France, 2014.

[31] M. Rodriguez-Blanco, A. Claudio-Sanchez, D. Theilliol, Vela-Valdes, L.G., P. Sibaja-Teran, L. HernandezGonzalez and J. Aguayo-Alquicira, "A Failure-detection strategy for IGBT based on gate-voltage behaviour applied to a motor drive system," IEEE Transactions on Industrial Electronics, vol. 58, no. 5, pp. 1625-1634, 2011.

[32] C. Lessmier, O. Enge-Rosenblatt, C. Bayer and D. Zimmer, "Data acquisition and signal analyssis from mesaured motor currents for defect detection in electromechanical drive systems," in European Conference of the Prognostics and Health Management Society 2014, Nantes, France, 2014.

[33] M. Rodriguez-Blanco, A. Vazquez-Perez, L. Hernandez-Gonzalez, V. Golikov, J. Aguayo-Alquicira and M. May-Alarcon, "Fault detection for IGBT using adaptive thresholds during the turn-on transient," IEEE Transactions on Industrial Electronics, vol. 62, no. 3, pp. 1975-1982, 2015.

[34] P. Ostojic, A. Banerjee, D. Patel, W. Basu and S. Ali, "Advanced motor monitoring and diagnostics," IEEE Transactions on Industry Applications, vol. 50, no. 5, pp. 3120-3129, 2014.

[35] P. Maggiore, M. D. L. Dall Vedova, L. Pace and A. Desando, "Definition of parametric methods for fault analysis applied to an electromechanical servomechanism affected by multiple failures," in European Conference of the Prognostics and Health Management Society 2014, Nantes, France, 2014.

[36] A. Widodo, B.-S. Yang, D.-S. Gu and B.-K. Choi, "Intelligent fault diagnosis system of induction motor based 
on transient current signal," Mechatronics, vol. 19, pp. 680-689, 2009.

[37] A. Bellazzi, G. Jacazio, B. Maino, G. Mihaylov and F. Pellerey, "A multivariate statistical approach to the implementation of a health monitoring system of mechanical power drive," in European Conference of the Prognostics and Health Management Society 2014, Nantes, France, 2014.

[38] p. Chandran, M. Lokesha, M. C. Majumder and K. Raheemv, "Application of Laplace wavelet kurtosis and wavelet statistical parameters for gear fault diagnosis," International journal of multidisciplinary sciences and engineering, vol. 3, no. 9, pp. 1-8, 2012.

[39] D. C. Montgomery, Introduction to Statistical Quality Control, 7th edition., New York: Wiley and Sons Inc., 2015.

[40] M. Reis and P. Saraiva, " Prediction of profiles in the process industries," Industrial \& Engineering Chemistry Research, vol. 51, pp. 4524-4266, 2012.

[41] W. H. Woodall, D. J. Sptizner, D. C. Montgomery and S. Gupta, "Using Control Charts to Monitor Process and Product Profiles," Journal of Quality Technology, vol. 36, no. 3, pp. 309-320, 2004.

[42] L. Kang and S. L. Albin, "On-line Monitoring When the Process Yields a Linear Profile," Journal of Quality Technology, vol. 32, pp. 418-426., 2000.

[43] M. Mahmoud, "Phase I analysis of multiple regressionlinear profiles," Communications in statistics, Simulation and Computation, vol. 37, no. 10, pp. 2106-2130, 2008.

[44] Y. Ding, L. Zeng and S. Zhou, "Phase I analysis for monitoring nonlinear profiles in manufacturing processes," Journal of Quality Technology, vol. 38, p. 199-216, 2006.

[45] A. Amiri, W. Jensen and R. Kazemzadeh, " A case study on monitoring polynomial profiles in the automotive industry," Quality \& Relibility Engineering International, vol. 26, p. 509-520, 2010.

[46] C.-H. Jen and S.-K. S. Fan, "Profile monitoring of reflow process using approximations of mixture secondorder polynomials," Journal of Chemometrics, vol. 28, pp. 815-833, 2014.

[47] S.-K. S. Fan, Y.-J. Chang and N. Aidara, "Nonlinear profile monitoring of reflow process data based on the sum of sine functions," Quality and Reliability ENgineering International, vol. 29, pp. 743-758, 2013.

[48] R. V. Brill, "A Case Study for Control Charting a Product Quality Measure That is a Continuous Function Over Time," in 45th Annual Fall Technical Conference, Toronto, Ontario, 2001.

[49] N. D. Tracy, J. Young and R. Mason, "Multivariate control charts for individual observations," Journal of Quality Technology, vol. 24, p. 88-95, 1992.

[50] D. Holmes and A. Mergen, "Improving the performance of the T2 Control Chart," Quality Engineering, vol. 5, p. 619-625, 1993.

[51] J. H. Sullivan and W. H. Woodall, "A Comparison of Multivariate quality control charts for individual observations," Journal of Quality Technology28, vol. 28, pp. 398-408, 1996.

[52] F. W. Scholz and T. J. Tosch, "Small Sample Uni-and Multivariate Control Charts for Means," in Proceedings of the American Statistical Association, Quality andProductivity Section, 1994.

[53] J. Williams, W. Woodall, J. Birch and J. Sullivan, "Distribution of Hotelling's T2 statistic based on the successive differences estimator," JournalQuality Technology 2006, vol. 38, no. 3, p. 217-229, 2006.

[54] N. Vargas, "Robust estimation in multivariate control charts for individual observations," Journal of Quality Technology, vol. 35, no. 4, pp. 367-376, 2003.

[55] D. M. Hawkins, "Regression adjustment for variables in multivariate quality control," Journal of Quality Technology, vol. 25, pp. 170-182, 1993.

[56] F. E. Grubbs, "Sample criteria for testing outlying observations," The Annals of Mathematical Statistics, vol. 21, no. 1, pp. 27-58, 1950. 
Appendix- I:

\begin{tabular}{|c|c|c|c|c|c|c|c|c|c|c|c|c|c|c|c|c|c|c|c|c|c|c|c|c|}
\hline \multirow[b]{2}{*}{ Time } & \multicolumn{4}{|c|}{ Explanatory Variables } & \multicolumn{14}{|c|}{ Phase I- Current (A) } & \multicolumn{6}{|c|}{ Phase II-Current (A) } \\
\hline & FR & DC & RPM & $\mathrm{P}$ & P1-H & $\mathrm{P} 2-\mathrm{H}$ & P3-H & $\mathrm{P} 4-\mathrm{H}$ & P5-H & P6-H & $\mathrm{P} 7-\mathrm{H}$ & $\mathrm{P8}-\mathrm{H}$ & P9-H & $\mathrm{P} 10-\mathrm{H}$ & $\mathrm{P} 11-\mathrm{H}$ & $\mathrm{P} 12-\mathrm{H}$ & $\mathrm{P} 13-\mathrm{H}$ & P14-H & P15-F & $\mathrm{P} 16-\mathrm{H}$ & P17-H & P18-H & P19-F & P20-F \\
\hline 1 & -0.2 & -1.0 & -0.2 & -0.9 & 4.75 & 5.14 & 3.81 & 4.84 & 4.05 & 5.33 & 4.15 & 5.20 & 5.15 & 4.54 & 4.80 & 4.23 & 4.80 & 5.26 & 2.75 & 5.07 & 5.30 & 3.58 & 4.01 & 3.97 \\
\hline 2 & -0.1 & -1.0 & -0.2 & -0.9 & 5.13 & 5.50 & 4.76 & 5.22 & 5.42 & 5.69 & 6.03 & 5.51 & 5.57 & 5.42 & 5.25 & 5.12 & 5.80 & 5.66 & 3.30 & 5.07 & 5.67 & 4.04 & 4.40 & 4.42 \\
\hline 3 & 0.0 & -1.0 & -0.1 & -0.9 & 5.31 & 5.63 & 4.87 & 5.16 & 5.39 & 5.88 & 5.94 & 4.92 & 6.13 & 4.78 & 5.43 & 5.15 & 6.80 & 5.85 & 3.23 & 4.80 & 5.72 & 4.23 & 4.49 & 4.50 \\
\hline 4 & 0.0 & -1.0 & -0.1 & -0.9 & 5.58 & 5.88 & 5.10 & 5.30 & 5.56 & 6.14 & 5.98 & 6.10 & 6.66 & 5.32 & 5.73 & 4.78 & 7.80 & 6.12 & 3.41 & 5.21 & 6.39 & 4.52 & 4.71 & 4.73 \\
\hline 5 & 0.1 & -1.0 & 0.0 & -0.9 & 5.75 & 6.04 & 5.30 & 5.29 & 5.54 & 6.30 & 6.20 & 6.30 & 6.80 & 5.78 & 5.88 & 4.94 & 8.80 & 6.29 & 3.36 & 5.16 & 6.31 & 4.67 & 4.80 & 4.81 \\
\hline 500 & -0.8 & -0.5 & -0.2 & 0.4 & 8.34 & 9.28 & 7.73 & 7.59 & 8.68 & 8.84 & 9.05 & 8.13 & 9.86 & 8.73 & 8.27 & 6.82 & 9.80 & 8.84 & 6.51 & 8.69 & 9.66 & 8.11 & 7.07 & 8.85 \\
\hline 501 & -0.8 & -0.5 & -0.2 & 0.4 & 8.34 & 8.81 & 8.14 & 7.60 & 8.21 & 8.84 & 8.42 & 7.89 & 9.47 & 7.99 & 8.27 & 6.91 & 10.80 & 8.85 & 6.51 & 8.52 & 8.88 & 8.11 & 7.07 & 8.85 \\
\hline 502 & -0.8 & -0.5 & -0.2 & 0.4 & 8.34 & 8.99 & 7.55 & 7.60 & 7.56 & 8.84 & 8.39 & 8.24 & 9.26 & 8.32 & 8.27 & 6.64 & 11.80 & 8.85 & 6.51 & 8.55 & 9.83 & 8.11 & 7.07 & 8.85 \\
\hline 1000 & -0.7 & -0.5 & -0.1 & 0.4 & 8.22 & 8.31 & 8.16 & 7.51 & 8.33 & 8.72 & 8.46 & 8.29 & 9.18 & 7.46 & 8.18 & 7.14 & 12.80 & 8.72 & 6.42 & 7.85 & 8.93 & 8.04 & 7.01 & 8.77 \\
\hline 1001 & -0.7 & -0.5 & -0.1 & 0.4 & 8.19 & 8.82 & 7.93 & 7.50 & 8.39 & 8.69 & 8.91 & 8.69 & 9.23 & 7.95 & 8.17 & 7.24 & 13.80 & 8.70 & 6.43 & 8.75 & 8.91 & 8.02 & 7.00 & 8.74 \\
\hline 2000 & -0.8 & -0.5 & -0.3 & 0.4 & 8.50 & 8.93 & 8.07 & 7.79 & 8.28 & 9.00 & 8.87 & 8.02 & 9.41 & 8.56 & 8.44 & 7.45 & 15.80 & 9.00 & 6.87 & 8.36 & 9.01 & 8.15 & 7.20 & 8.91 \\
\hline 2001 & -0.8 & -0.5 & -0.3 & 0.4 & 8.67 & 9.25 & 8.43 & 7.93 & 9.29 & 9.17 & 10.27 & 8.43 & 10.14 & 9.23 & 8.58 & 7.35 & 16.80 & 9.17 & 7.05 & 8.69 & 9.66 & 8.24 & 7.31 & 9.02 \\
\hline 3000 & -0.9 & -0.5 & -0.5 & 0.5 & 9.26 & 9.88 & 9.13 & 8.38 & 9.78 & 9.77 & 10.40 & 8.79 & 10.05 & 9.60 & 9.07 & 7.66 & 18.80 & 9.76 & 7.47 & 9.80 & 10.46 & 8.66 & 7.70 & 9.47 \\
\hline 3001 & -0.9 & -0.5 & -0.5 & 0.5 & 9.28 & 9.75 & 8.84 & 8.39 & 9.57 & 9.78 & 10.29 & 8.81 & 10.05 & 8.90 & 9.08 & 7.41 & 19.80 & 9.77 & 7.48 & 8.89 & 10.63 & 8.66 & 7.71 & 9.48 \\
\hline 4000 & -0.9 & -0.5 & -0.6 & 0.4 & 9.71 & 10.13 & 8.89 & 8.84 & 8.99 & 10.21 & 9.64 & 9.55 & 10.30 & 9.24 & 9.52 & 8.78 & 21.80 & 10.20 & 8.08 & 10.01 & 10.42 & 8.94 & 8.08 & 9.78 \\
\hline 4001 & -0.9 & -0.5 & -0.6 & 0.4 & 9.57 & 9.76 & 8.74 & 8.71 & 9.44 & 10.07 & 10.17 & 9.82 & 10.53 & 9.57 & 9.39 & 8.54 & 22.80 & 10.06 & 7.93 & 9.66 & 10.75 & 8.84 & 7.97 & 9.67 \\
\hline 5000 & -0.9 & -0.5 & -0.5 & 0.4 & 9.40 & 9.87 & 9.24 & 8.66 & 9.74 & 9.91 & 10.68 & 9.67 & 10.00 & 9.70 & 9.28 & 8.22 & 24.80 & 9.90 & 7.95 & 9.75 & 10.36 & 8.70 & 7.90 & 9.53 \\
\hline 5001 & -0.9 & -0.5 & -0.5 & 0.4 & 9.41 & 10.26 & 9.25 & 8.66 & 9.95 & 9.91 & 10.68 & 8.94 & 10.88 & 9.80 & 9.29 & 8.62 & 25.80 & 9.90 & 7.96 & 9.61 & 11.09 & 8.70 & 7.90 & 9.53 \\
\hline 6000 & -0.8 & -0.5 & -0.4 & 0.4 & 8.73 & 9.05 & 8.42 & 8.06 & 8.94 & 9.23 & 9.15 & 8.23 & 9.67 & 8.66 & 8.68 & 7.14 & 27.80 & 9.23 & 7.28 & 8.68 & 9.19 & 8.25 & 7.39 & 9.04 \\
\hline 6001 & -0.8 & -0.5 & -0.3 & 0.4 & 8.66 & 8.91 & 8.63 & 7.99 & 9.05 & 9.16 & 9.34 & 8.19 & 9.10 & 7.95 & 8.61 & 7.40 & 28.80 & 9.16 & 7.19 & 8.27 & 9.52 & 8.21 & 7.34 & 8.99 \\
\hline 7000 & -0.7 & -0.5 & -0.2 & 0.4 & 8.09 & 8.80 & 7.83 & 7.55 & 8.77 & 8.59 & 9.46 & 8.15 & 9.06 & 8.02 & 8.17 & 7.21 & 30.80 & 8.60 & 6.74 & 8.18 & 9.44 & 7.86 & 6.99 & 8.58 \\
\hline 7001 & -0.7 & -0.5 & -0.2 & 0.4 & 8.02 & 8.95 & 7.04 & 7.49 & 7.06 & 8.52 & 7.20 & 8.45 & 9.51 & 8.89 & 8.12 & 7.05 & 31.80 & 8.52 & 6.68 & 8.58 & 9.92 & 7.82 & 6.94 & 8.53 \\
\hline 8000 & -0.7 & -0.5 & -0.2 & 0.3 & 7.96 & 8.76 & 7.89 & 7.49 & 8.33 & 8.46 & 8.70 & 8.27 & 9.57 & 8.57 & 8.10 & 7.12 & 33.80 & 8.47 & 6.75 & 8.25 & 8.96 & 7.76 & 6.93 & 8.47 \\
\hline 8001 & -0.7 & -0.5 & -0.2 & 0.3 & 8.02 & 8.88 & 7.61 & 7.54 & 7.69 & 8.52 & 8.14 & 8.09 & 9.34 & 8.12 & 8.15 & 6.97 & 34.80 & 8.52 & 6.81 & 8.35 & 9.55 & 7.79 & 6.96 & 8.50 \\
\hline 9000 & -0.6 & -0.5 & 0.0 & 0.4 & 7.74 & 8.26 & 7.56 & 7.26 & 7.60 & 8.24 & 7.70 & 8.20 & 9.22 & 7.88 & 7.92 & 6.87 & 36.80 & 8.25 & 6.35 & 8.07 & 8.99 & 7.71 & 6.80 & 8.39 \\
\hline 9001 & -0.7 & -0.5 & 0.0 & 0.4 & 7.81 & 8.20 & 7.70 & 7.29 & 8.01 & 8.31 & 8.18 & 8.21 & 8.96 & 7.44 & 7.96 & 6.45 & 37.80 & 8.32 & 6.35 & 7.75 & 9.08 & 7.77 & 6.83 & 8.45 \\
\hline 10000 & -0.6 & -0.5 & 0.0 & 0.3 & 7.59 & 7.53 & 7.34 & 7.18 & 7.75 & 8.09 & 8.12 & 8.07 & 8.41 & 7.46 & 7.85 & 6.65 & 39.80 & 8.10 & 6.34 & 7.42 & 8.03 & 7.61 & 6.75 & 8.27 \\
\hline
\end{tabular}


Appendix II

\begin{tabular}{ccccccccccccc}
\hline & $\beta_{0}$ & $\beta_{1}$ & $\beta_{2}$ & $\beta_{3}$ & $\beta_{4}$ & $\beta_{11}$ & $\beta_{22}$ & $\beta_{44}$ & $\beta_{13}$ & $\beta_{14}$ & $\beta_{23}$ & $\beta_{34}$ \\
\hline$\beta_{0}$ & 1.83 & -0.17 & 0.17 & -0.44 & 0.04 & -0.82 & 0.07 & 0.12 & 0.02 & -0.51 & -0.11 & -0.58 \\
$\beta_{1}$ & -0.17 & 1.09 & -0.09 & 0.10 & 0.44 & -0.13 & -0.08 & -0.07 & 0.00 & -0.06 & -0.17 & 0.10 \\
$\beta_{2}$ & 0.17 & -0.09 & 0.12 & -0.15 & -0.21 & 0.12 & 0.03 & -0.03 & -0.01 & -0.21 & 0.01 & -0.07 \\
$\beta_{3}$ & -0.44 & 0.10 & -0.15 & 0.82 & 0.04 & -0.59 & -0.04 & 0.06 & 0.03 & 0.35 & -0.05 & 0.12 \\
$\beta_{4}$ & 0.04 & 0.44 & -0.21 & 0.04 & 1.01 & 0.01 & -0.02 & 0.32 & 0.10 & 0.38 & -0.04 & 0.12 \\
$\beta_{11}$ & -0.82 & -0.13 & 0.12 & -0.59 & 0.01 & 1.81 & 0.03 & 0.09 & -0.04 & -0.06 & 0.11 & 0.49 \\
$\beta_{22}$ & 0.07 & -0.08 & 0.03 & -0.04 & -0.02 & 0.03 & 0.02 & 0.01 & 0.03 & -0.01 & 0.02 & -0.03 \\
$\beta_{44}$ & 0.12 & -0.07 & -0.03 & 0.06 & 0.32 & 0.09 & 0.01 & 0.44 & -0.14 & 0.03 & 0.01 & 0.15 \\
$\beta_{13}$ & 0.02 & 0.00 & -0.01 & 0.03 & 0.10 & -0.04 & 0.03 & -0.14 & 0.34 & 0.20 & -0.01 & -0.22 \\
$\beta_{14}$ & -0.51 & -0.06 & -0.21 & 0.35 & 0.38 & -0.06 & -0.01 & 0.03 & 0.20 & 0.76 & 0.07 & 0.02 \\
$\beta_{23}$ & -0.11 & -0.17 & 0.01 & -0.05 & -0.04 & 0.11 & 0.02 & 0.01 & -0.01 & 0.07 & 0.07 & 0.02 \\
$\beta_{34}$ & -0.58 & 0.10 & -0.07 & 0.12 & 0.12 & 0.49 & -0.03 & 0.15 & -0.22 & 0.02 & 0.02 & 0.50 \\
\hline
\end{tabular}

